\title{
Kinetic switching between two modes of bisurea surfactant self-assembly $\dagger$
}

\author{
Matthijn R. J. Vos, ${ }^{* a b}$ Philippe E. L. G. Leclère, $\ddagger^{c}$ Hugo Meekes, ${ }^{d}$ Elias Vlieg, ${ }^{d}$ \\ Roeland J. M. Nolte ${ }^{d}$ and Nico A. J. M. Sommerdijk $* a$
}

Received 15th April 2010, Accepted 5th July 2010

DOI: $10.1039 / \mathrm{c0cc00967a}$

The size and shape of a surfactant aggregate could be altered by using supersaturation like in crystal growth, rather than applying common laws that drive surfactant aggregate formation.

Surfactant assemblies display a large variety of morphologies with different shapes and sizes, such as micelles, vesicles, rods, tubules and sheets. ${ }^{1-3}$ As several of such assemblies are promising as materials for various biomedical applications, ${ }^{4}$ it is important to obtain control over the morphology and the dimensions of these self-assembled objects. ${ }^{5}$ The use of mechanical forces, ${ }^{6}$ the mixing of different surfactants, ${ }^{7}$ or the use of additives ${ }^{8}$ have been applied to achieve morphological control over the resulting architectures. In the absence of strong intermolecular forces the aggregate morphology can be predicted from the molecular structure using the postulates of Israelachvili. ${ }^{2}$ However, when specific supramolecular interactions such as hydrogen bonds are involved the factors determining the size and shape of the assembly are not fully understood.

Previously, we introduced a bis-ureido based surfactant 1, which, due to strong directional hydrogen bonding interactions, forms well-defined highly ordered ribbon-like aggregates in water. ${ }^{9}$ These aggregates had a thickness of $6 \mathrm{~nm}$, widths of $70 \pm 20 \mathrm{~nm}$ and lengths in the micrometre regime $\S$ with almost no variation in width over the entire length of a single ribbon. Here we show that due to a combination of hydrophobic forces and strong directional hydrogen bonding interactions the assemblies of $\mathbf{1}$ do not behave like a micellar system, but in fact are 2D crystal-like structures. The formation of ribbons and tubes of different shapes and sizes is known for e.g. phospholipids and gemini-surfactants, however control over the shape and size has been only reported by variations in molecular structure or by changing the ratio of mixed surfactant systems. ${ }^{10}$ In the system discussed here we tune the size and morphology of the aggregates only by changing

\footnotetext{
${ }^{a}$ Laboratory of Materials and Interface Chemistry and Soft Matter Cryo-TEM Research Unit, Eindhoven University of Technology, Eindhoven, The Netherlands. E-mail: n.sommerdijk@tue.nl, matthijn.vos@fei.com; Fax: +31 40245 1036;

Tel: +31402475870

${ }^{b}$ FEI Company, Achtseweg Noord 5, 5600 KA Eindhoven,

The Netherlands

${ }^{c}$ University of Mons (UMons), Laboratory for Chemistry of Novel Materials, Center for Innovation and Research in Materials and Polymers (CIRMAP), Place du Parc, 20 B-7000 Mons, Belgium

${ }^{d}$ Radboud University Nijmegen, Institute for Molecules and Materials, Heyendaalseweg 135, 6525 AJ Nijmegen, The Netherlands

$\dagger$ Electronic supplementary information (ESI) available: Experimental details, synthetic procedures and additional microscopy images. See DOI: $10.1039 / \mathrm{c} 0 \mathrm{cc} 00967 \mathrm{a}$

$\ddagger$ Ph.L. is a Research Associate from FRS-FNRS, Belgium.
}

the supersaturation of the solution. In addition two different modes of assembly could be discerned.

Aggregates of surfactant 1 were prepared by heating an aqueous suspension to $90{ }^{\circ} \mathrm{C}$ and subsequent cooling to $\mathrm{RT}$ yielding a viscous, milky suspension. Cryo-TEM investigation showed that upon increasing the concentration from 0.25 to $10 \mathrm{mg} \mathrm{ml}^{-1}$ the width of the ribbons in freshly prepared solutions decreased (Fig. 1). In addition, as the widths of the ribbons decreased, the corresponding size distribution also became narrower. This contrasts the behavior generally observed for micellar systems, in which the size of the aggregates and their size distribution increases when the concentration is increased. ${ }^{2}$ In fact, the observed narrowing of the ribbons formed at higher concentration shows an analogy to crystal growth, with a higher nucleation rate at higher surfactant concentrations resulting in a smaller average width and, concomitantly, a narrower size distribution.

These results triggered us to investigate the degree of tuning that can be obtained when this surfactant system is treated as a crystal rather than a surfactant aggregate. We assumed that these aggregates can grow in time like a crystal, rather than be formed immediately like in micellar- and vesicile-like system.

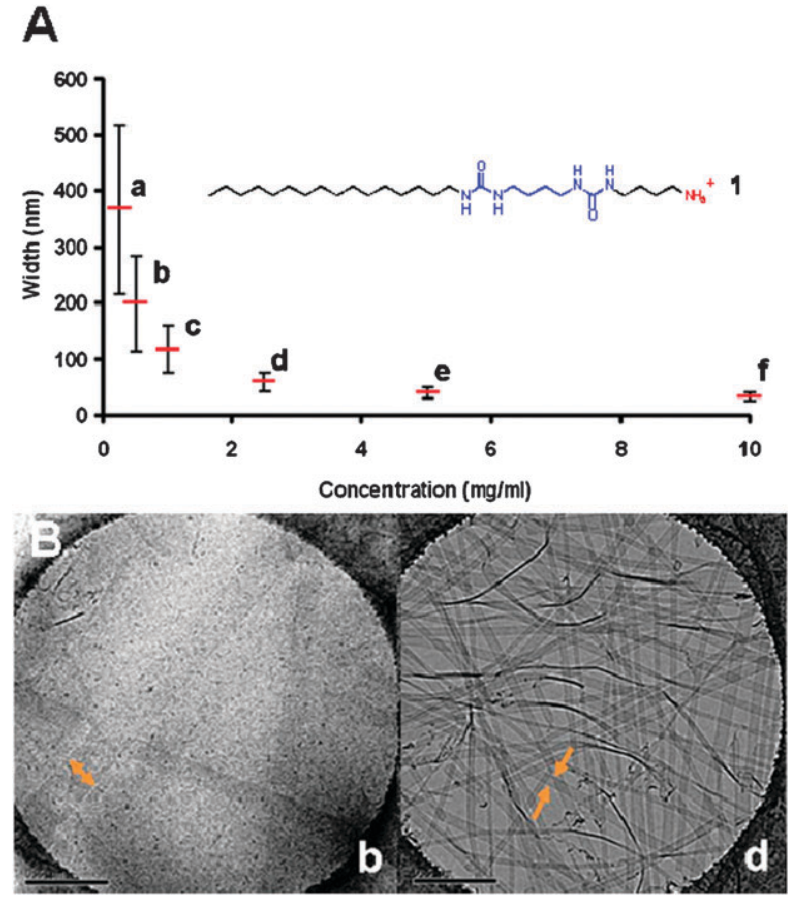

Fig. 1 (A) Plot of the ribbon width $v s$. the surfactant concentration (a: 0.25 , b: 0.5 , c: $1.0, \mathrm{~d}: 2.5$, e: $5.0, \mathrm{f}: 10.0 \mathrm{mg} \mathrm{m}^{-1}$ ). (B) Cryo-TEM examples of conc. b and d, clearly indicating width narrowing; scale bars $500 \mathrm{~nm}$. 
An assembly mechanism similar to that found in crystal growth implies a dense 2-dimensional crystal-like packing of the surfactant molecules. Previously, ${ }^{9}$ we described the structure of the aggregates of $\mathbf{1}$ as ribbons consisting of an interdigitated double layer of close-packed surfactant molecules that are interconnected by chains of hydrogen bonds running along the length of the ribbon. With additional structural information we have refined this structure (see $\mathrm{ESI} \dagger$ ).

We speculated that, by allowing the ribbon aggregates to grow more slowly, the growth in the fastest growing direction (along the $y$-axis) would be most affected, thereby resulting in aggregates with a smaller aspect ratio (a.r.: length/width) and thus not only controlling their width, as could be achieved when changing the concentration, but also their length. To this end we studied the development of the aggregates just below the formation temperature $70{ }^{\circ} \mathrm{C}$ (see $\mathrm{ESI} \dagger$ ).

The growth of aggregates was performed using $0.25 \mathrm{mg} \mathrm{ml}^{-1}$ surfactant solution which was prepared above the formation temperature in a closed vial by heating to $90{ }^{\circ} \mathrm{C}$. Subsequently, the aggregates were allowed to grow by keeping the solution at $65{ }^{\circ} \mathrm{C}$ for 1 week. AFM and TEM showed the formation of faceted $6 \mathrm{~nm}$ thick crystal-like aggregates whose average aspect ratio was 2.5 ; a dramatic decrease compared to the ribbons grown at room temperature (a.r. $>50$ ). Irrespective of their size almost all of these aggregates showed faceted top ends with an average top angle of $125^{\circ}(N=80)$ reflecting their organization on a molecular level (see ESI $\dagger$ for more detail).

In order to further investigate the level of tuning that can be achieved over the aspect ratios, the aggregates were grown at different temperatures between 25 and $68{ }^{\circ} \mathrm{C}$. Strikingly, the aggregates formed below $55^{\circ} \mathrm{C}$ were all ribbons (i.e. aggregates with a.r. $>50$ ), whereas those formed at higher temperatures displayed crystal-like morphologies. The high lengths of the ribbons caused gelation of the samples, and hampered the accurate determination of their aspect ratios.

Nevertheless a decrease in ribbon length was apparent for those that were grown at higher temperatures (between $45-55^{\circ} \mathrm{C}$ ). For the aggregates grown at temperatures $>55{ }^{\circ} \mathrm{C}$ a clear decrease in length was observed at increasing temperatures and, interestingly, also the distribution in lengths became smaller at higher temperature. In contrast, no proportional effect was observed on the widths of the aggregates, which varied between 200 and $850 \mathrm{~nm}$ at all temperatures (Fig. 2A and $\mathrm{C}$ ). The apparent decrease in aspect ratio of the aggregates found at higher temperatures is therefore mostly due to a decrease in length. The faceted top ends were found to be present for all crystal-like aggregates, but the $125^{\circ}$ angles were better defined for those grown at the highest temperatures. Close examination of many top ends from ribbons grown at the lower temperatures, where the ribbon morphology dominates, revealed slanted, rounded, fragmented and unfinished ends. In addition, in some cases dendritic and dagger-shaped structures were observed (Fig. 3).

The change in morphology and the formation of facets indicate a different growth mechanism at higher temperatures compared to the one at lower temperatures by which ribbons are formed. To investigate this further, first the thermal behavior was analysed in detail.
A
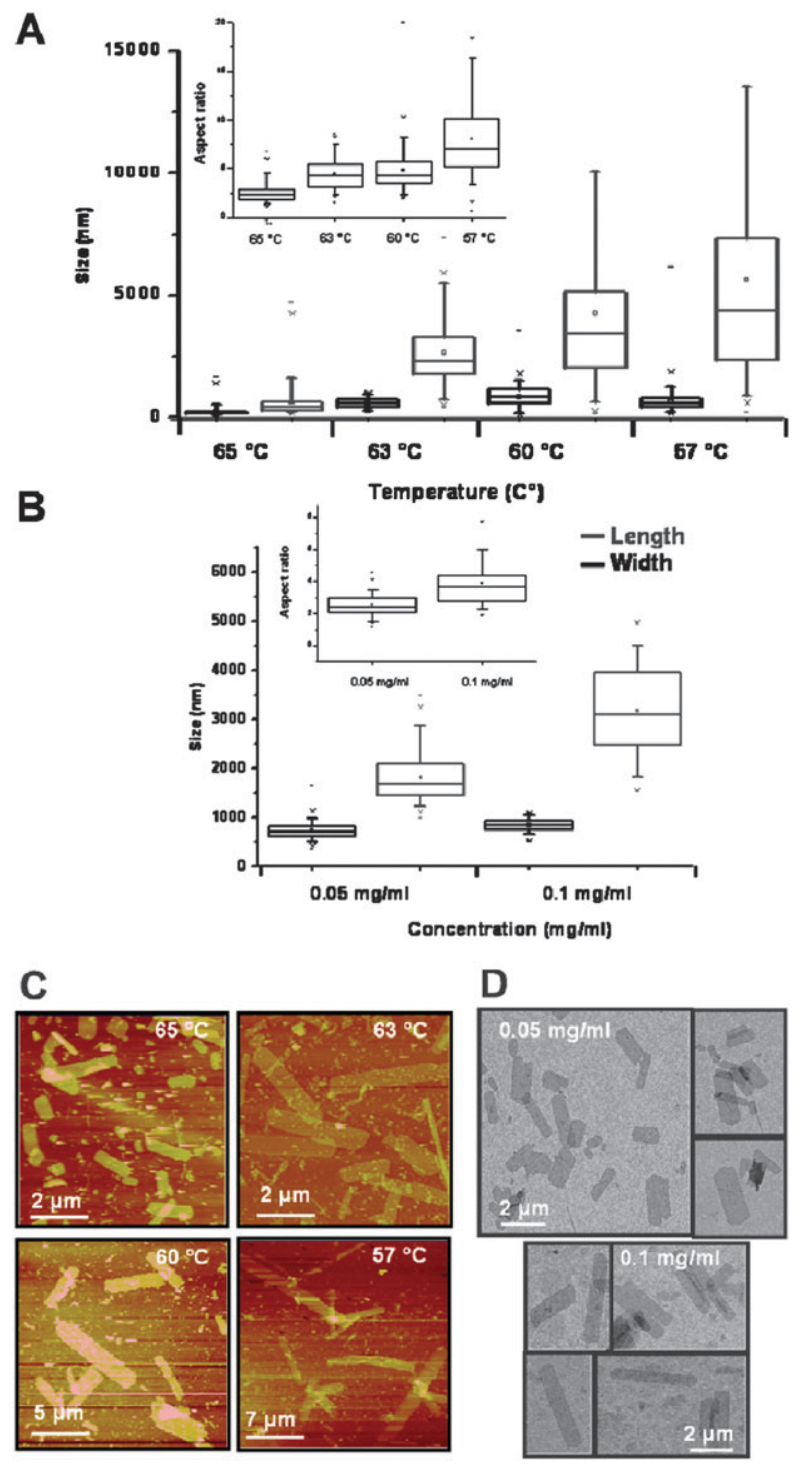

Fig. 2 (A) Box-whisker plot of the length and width distribution of the 2-D crystal-like morphology as a function of temperature (for each temperature $N>100$ ). (B) Same as A only as a function of concentration (for each concentration $N>100$ ). (C)/(D) Corresponding representative AFM $\backslash$ TEM images of $\mathrm{A}$ and $\mathrm{B}$.

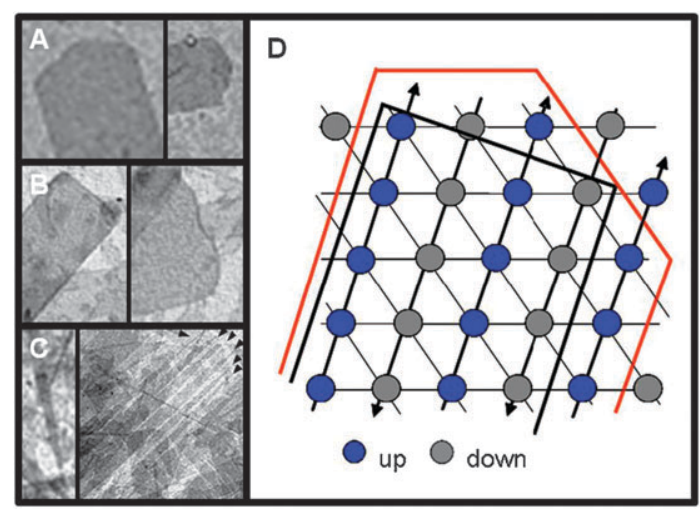

Fig. 3 Variety of top-end morphology: (A) faceted, (B) strait, (C) needle and dendritic, (D) crystal-lattice/-planes. 
Differential scanning calorimetry (DSC) of a concentrated gel $\left(12.8 \mathrm{mg} \mathrm{ml}^{-1}\right)$ showed two endotherms: a small $-0.34 \mathrm{~kJ} \mathrm{~mol}^{-1}$ peak (onset at $67.7{ }^{\circ} \mathrm{C}$, max. $69.5^{\circ} \mathrm{C}$ ) and a larger $-2.24 \mathrm{~kJ} \mathrm{~mol}^{-1}$ peak (onset $72.9{ }^{\circ} \mathrm{C}$, max. $75.6{ }^{\circ} \mathrm{C}$ ). These endotherms are tentatively attributed to the melting of the alkyl tail segment followed by the total disintegration of the ribbon aggregates and inter-molecular hydrogen bond breakage. VT-IR spectroscopy showed no significant changes in the $\mathrm{CH}_{2}$ symmetric $\left(2849 \mathrm{~cm}^{-1}\right)$ and asymmetric (2921 $\mathrm{cm}^{-1}$ ) stretch vibrations, nor in the $\mathrm{C}=\mathrm{O}$ stretch vibration $\left(1602 \mathrm{~cm}^{-1}\right)$ between $20-70{ }^{\circ} \mathrm{C}$, indicating that no changes in the molecular packing occurred in this temperature interval. In addition, the position of the $\mathrm{CH}_{2}$-deformation band can be used to assess the organization of the hydrocarbon chains. Gauche conformations give rise to a band at low wavenumbers $\left(\sim 1463 \mathrm{~cm}^{-1}\right)$, whereas a highly packed all-trans state leads to a vibration at $1471 \mathrm{~cm}^{-1}$. The observed position of the deformation band $\left(1471 \mathrm{~cm}^{-1}\right)$ indeed indicated an all-trans conformation, which upon heating to $70{ }^{\circ} \mathrm{C}$ showed no shift to lower wavenumbers. Only above $70{ }^{\circ} \mathrm{C}$ a broadening of the signals was observed. This was attributed to the melting of the alkyl chain segment and concomitant dissolution of the ribbons, in agreement with the results from DSC, implying that the hydrophobic forces play an essential role in the stabilization of the aggregates.

As VT-FTIR indicated, no significant changes in the hydrogen bonding interactions within the ribbons occurred upon increasing the temperature. The differences in aggregate morphology found at different growth temperatures were therefore tentatively attributed to kinetic effects associated with changes in supersaturation level.

In order to generate the $2 \mathrm{D}$ crystal-like morphology also using concentration and verify this hypothesis, fresh samples were prepared at room temperature with lower surfactant concentrations as in Fig. $1\left([\mathbf{1}]=0.05\right.$ and $\left.0.1 \mathrm{mg} \mathrm{ml}^{-1}\right)$. Conventional TEM revealed the formation of faceted aggregates with shorter lengths for those grown at $0.05 \mathrm{mg} \mathrm{ml}^{-1}$ compared to those formed at $0.1 \mathrm{mg} \mathrm{ml}^{-1}$ (Fig. 2B and D). This shows that the aggregate morphology can be changed in the same fashion using both temperature and concentration supporting the idea that this effect is driven by the supersaturation rather than by the $\mathrm{H}$-bonding strength, which is only controlled by temperature.

The strong H-bonds are responsible for the high growth rate in the length direction. Apart from the hydrophobic interactions an extra $\mathrm{H}$-bond can be formed in this direction and this direction is therefore likely to grow faster, since the other directions are only stabilized by hydrophobic interactions alone. In crystal growth, facets will disappear in the fastest growth direction and only the slowest growing crystal planes will determine the final morphology. Reducing the supersaturation to such an extent that the crystal-like morphology dominates is apparently sufficient to show faceted growth even in the length direction causing the 2D crystal-like morphology.
However, when the supersaturation is increased the growing speed in the fastest growth direction is most affected causing disappearance of the facets, roughening effects (Fig. 3) and transport limited growth, like in 3D crystal growth (see ESI $\dagger$ ).

In summary we have demonstrated that bis-urea amphiphile 1 assembles in bilayer aggregates of which the morphology and aspect ratio can be controlled through variation of the supersaturation at which they are formed, by means of the temperature and concentration of the solution. By increasing the surfactant concentration an elongation of the aggregates was observed combined with a switch from a ribbon-like appearance at high supersaturation to a $2 \mathrm{D}$ crystal-like appearance at low supersaturation levels of the aggregates. By influencing the kinetics of the growth process we were able to select the morphology of the aggregates.

\section{Notes and references}

$\S$ Due to the large length, quantitative determination of the length size was hampered.

1 J.-H. Fuhrhop and W. Helfrich, Chem. Rev., 1993, 93, 1565.

2 J. N. Israelachvili, Intermolecular and Surface Forces, Academic Press, London, 2nd edn, 1992.

3 J. A. A. Elemans, A. E. Rowan and R. J. M. Nolte, J. Mater. Chem., 2003, 13, 2661; M. C. Feiters and R. J. M. Nolte, Chiral Self-assembled Structures of Biomolecules and Synthetic Analogues, in Advances in Supramolecular Chemistry, ed. G. W. Gokel, Jai Press Inc., Stamford, CT, USA, 2000, vol. 6, pp. 41-156.

4 J. D. Hartgerink, E. Beniash and S. I. Stupp, Science, 2001, 294, 1684; E. Beniash, J. D. Hartgerink, H. Storrie, J. C. Stendahl and S. I. Stupp, Acta Biomater., 2005, 1, 387; M. O. Guler, S. Soukasene, J. F. Hulvat and S. I. Stupp, Nano Lett., 2005, 5, 249; K. L. Niece, J. D. Hartgerink, J. J. J. M. Donners and S. I. Stupp, J. Am. Chem. Soc., 2003, 125, 7146.

5 S. I. Stupp, M. U. Pralle, G. N. Tew, L. Li, M. Sayar and E. R. Zubarev, MRS Bull., 2000, 25, 42.

6 K. Sou, Y. Naito, T. Endo, S. Takeoka and E. Tsuchida, Biotechnol. Prog., 2003, 19, 1547; M. Antonietti and S. Förster, Adv. Mater. 2003, 15, 1323; S. Rasi, F. Mavelli and P. L. Luisi, J. Phys. Chem. B, 2003, 107, 14068; S. Wen, M. Helmstedt and H. J. Knoll, J. Colloid Interface Sci., 2004, 278, 488; S. Chungcharoenwattana, H. Kashiwagi and M. Ueno, Colloid Polym. Sci., 2005, 283, 1180; S. Chungcharoenwattana and M. Ueno, Chem. Pharm. Bull., 2004, 52, 1058 .

7 H.-T. Jung, S. Y. Lee, E. W. Kaler, B. Coldren and J. A. Zasadzinski, Proc. Natl. Acad. Sci. U. S. A., 2002, 99, 15318; S. Nilsson, K. Thuresson, P. Hansson and B. Lindman, J. Phys. Chem. B, 1998, 102, 7099; O. Söderman, K. L. Herrington, E. W. Kaler and D. D. Miller, Langmuir, 1997, 13, 5531; A. Kahn and E. Marques, Specialist Surfactants, Chapman and Hall, London, 1996.

8 N. Heldt, J. Zhao, S. Friberg, Z. Zhang, G. Slack and Y. Li, Tetrahedron, 2000, 56, 6985; O. Uzun, A. Sanyal, H. Nakade, R. J. Thibault and V. M. Rotello, J. Am. Chem. Soc., 2004, 126, 14773; A. Moschetta, P. M. Frederik, P. Portincasa, G. P. VanBerge-Henegouwen and K. J. Van Erpecum, J. Lipid Res., 2002, 43, 1046; A. Walter, P. K. Vinson, A. Kaplun and Y. Talmon, Biophys. J., 1991, 60, 1315; P. M. Frederik and N. Sommerdijk, Curr. Opin. Colloid Interface Sci., 2005, 10, 245.

9 M. R. J. Vos, G. Etxebarria Jardí, A. Llanes Pallas, M. Breurken, O. L. J. van Asselen, P. H. H. Bomans, P. E. L. G. Leclère, P. M. Frederik, R. J. M. Nolte and N. A. J. M. Sommerdijk, J. Am. Chem. Soc., 2005, 127(16), 768.

10 A. Singh, E. M. Wong and J. M. Schnur, Langmuir, 2003, 19, 1888. 